\title{
Consumption of energy-dense diets in relation to metabolic syndrome and inflammatory markers in Iranian female nurses
}

\author{
Leila Azadbakht 1,2,3,4, Fahimeh Haghighatdoost ${ }^{5}$, Ammar Hassanzadeh Keshteli $^{6}$, \\ Bagher Larijani ${ }^{7}$ and Ahmad Esmaillzadeh ${ }^{8,2,3,4, *}$
}

'Diabetes Research Center, Endocrinology and Metabolism Clinical Sciences Institute, Tehran University of Medical Sciences, Tehran, Islamic Republic of Iran: ${ }^{2}$ Department of Community Nutrition, School of Nutritional Sciences and Dietetics, Tehran University of Medical Sciences, Tehran, Islamic Republic of Iran: ${ }^{3}$ Food Security Research Center, School of Nutrition and Food Science, Isfahan University of Medical Sciences, Isfahan, Islamic Republic of Iran: ${ }^{4}$ Department of Nutrition, School of Nutrition and Food Science, Isfahan University of Medical Sciences, Isfahan, Islamic Republic of Iran: ${ }^{5}$ Department of Community Nutrition, School of Nutrition and Food Science, Isfahan University of Medical Sciences, Isfahan, Islamic Republic of Iran: ${ }^{6}$ Department of Medicine, University of Alberta, Edmonton, Alberta, Canada: ${ }^{7}$ Endocrinology and Metabolism Research Center, Endocrinology and Metabolism Clinical Sciences Institute, Tehran University of Medical Sciences, Tehran, Islamic Republic of Iran: ${ }^{8}$ Obesity and Eating Habits Research Center, Endocrinology and Metabolism Molecular-Cellular Sciences Institute, Tehran University of Medical Sciences, PO Box 14155/6117, Tehran, Islamic Republic of Iran

Submitted 2 April 2016: Final revision received 31 August 2016: Accepted 2 September 2016: First published online 16 November 2016

\begin{abstract}
Objective: To examine the relationship between dietary energy density (DED) and risk of metabolic syndrome (MetS), its components and inflammatory markers. Design: Cross-sectional study. Dietary intakes were assessed using a validated dish-based semi-quantitative FFQ. DED was calculated by dividing energy intake $(\mathrm{kcal} / \mathrm{d})$ by the total weight of foods only $(\mathrm{g} / \mathrm{d})$. MetS was defined based on the National Cholesterol Education Program Adult Treatment Panel III criteria. All associations were examined in the quartiles of DED, with higher quartiles indicating more energy-dense diets.

Setting: Isfahan, Iran.

Subjects: Female nurses ( $n$ 1036) aged $>30$ years.

Results: After controlling for potential confounders, individuals in the top quartile of DED had $78 \%$ greater chance of MetS compared with those in the first $(\mathrm{OR}=1.78 ; 95 \%$ CI 1.36, 2.98; $P<0.001)$. Individuals in the highest quartile of DED were more likely to be abdominally obese (OR $=1.51 ; 95 \%$ CI 1.00, 2.63) and have hypertriacylglycerolaemia $(\mathrm{OR}=2.95 ; 95 \% \mathrm{CI} 1.58,3.91)$ and low HDL cholesterol levels (OR $=1.36$; 95\% CI 1.17, 2.54) compared with those in the lowest quartile. Mean concentration of plasma high-sensitivity C-reactive protein (hs-CRP) across increasing quartiles of DED was $1.7,1 \cdot 7,2 \cdot 0,2.4 \mathrm{mg} / 1$ ( $P$ for trend $=0.04)$. Such increasing concentrations across increasing quartiles of DED were also seen for TNF- $\alpha(4 \cdot 1,4 \cdot 5,4.5,4.8 \mathrm{ng} / \mathrm{l} ; P$ for trend=0.03) and IL-6 (1.6, 1.6, 1.5, $2 \cdot 5 \mathrm{ng} / \mathrm{l} ; P$ for trend $<0 \cdot 01)$.

Conclusions: Consumption of high-energy-dense foods was associated with increased chance of MetS, most of its features and inflammatory markers including hs-CRP, TNF- $\alpha$ and IL-6.
\end{abstract}

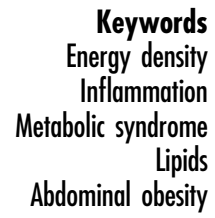

Metabolic syndrome (MetS), as a clustering of metabolic abnormalities, is a well-known risk factor for CVD, type 2 diabetes and early mortality. The growing trend in the prevalence of MetS is a global health problem in both developed and developing nations ${ }^{(1)}$. Several dietary and non-dietary factors have been involved in the pathogenesis of MetS. Among others, obesity, unhealthy food intake, lack of physical activity and smoking contribute extensively to these conditions ${ }^{(2)}$. Elevated concentrations of inflammatory biomarkers have also been suggested as a possible mediating mechanism through which dietary intakes and obesity might affect the risk of chronic disorders.

Dietary energy density (DED) is defined as the amount of energy per unit weight of food ${ }^{(3)}$. High-energy-dense diets are associated with higher energy intake and weight 
gain $^{(4-9)}$. Excess body weight and fat mass are linked to greater inflammatory markers and chronic diseases. High-energy-dense diets are characterized mainly by low consumption of fresh fruits and vegetables but high consumption of fats and refined carbohydrates ${ }^{(10,11)}$. Although the association of dietary intakes with metabolic abnormalities has frequently been examined, data on the association of DED with chronic diseases are scarce. Examining the association of DED with chronic diseases could be more reliable than single dietary components, since it accounts for the enormous interactions between nutrients and foods.

Available evidence assessing the association between DED and risk of MetS is limited and inconsistent. While some epidemiological investigations indicated a positive link between DED and the odds of diabetes, insulin resistance, MetS and abdominal adiposity ${ }^{(10,12,13)}$, others failed to find such significant relationships ${ }^{(4,14)}$. Besides cardiometabolic abnormalities, few studies have examined the association of DED with inflammation ${ }^{(4,7,15)}$. Despite a slight decrement in serum concentrations of C-reactive protein (CRP) following the consumption of a low-energydense diet in a short-term clinical trial ${ }^{(7)}$, findings from a large population-based study and an 8-week clinical trial revealed no significant correlation between DED and serum high-sensitivity (hs)-CRP or other inflammatory adipokines $^{(4,15)}$.

It must be kept in mind that previous studies on dietinflammation-CVD associations were mostly conducted in Western countries and limited data are available in this regard in the understudied region of the Middle East. Different dietary patterns and lifestyle in this part of the world might reveal different associations between energy density and these conditions. Due to low intakes of fruits and vegetables and high intake of refined grains, Middle Eastern populations consume more energy-dense diets than Europeans ${ }^{(10,11)}$. Therefore, assessing the association between DED and these cardiometabolic abnormalities is particularly relevant in these populations. We hypothesized that higher DED is associated with increased risk of MetS as well as higher levels of inflammatory biomarkers, and conducted the present study to identify the association of DED with inflammation and MetS in a large group of Iranian female nurses.

\section{Materials and methods}

\section{Participants}

The current cross-sectional study was conducted in a representative sample of Isfahani female nurses aged $>30$ years. To perform random sampling, we provided a list of all hospitals (both public and private) in different parts of Isfahan. Then, we randomly selected some hospitals considering the number of private and public hospitals in each area ( $n$ 12). Nurses in the selected hospitals were listed and then a random sample of nurses in each hospital was chosen based on the number of nurses working in that hospital. Totally, 1102 nurses of 1196 invited nurses agreed to participate in our study. We excluded individuals who had a prior history of CVD, diabetes, cancer and stroke ( $n$ 11), or infection ( $n 3)$. In addition, those who had left at least seventy items blank on the FFQ ( $n 12)$, those who reported a total daily energy intake outside the range of 3347-17 573 kJ (800-4200 kcal; $n$ 29) and those who were taking medications that affect glucose homeostasis ( $n$ 11) were excluded. After these exclusions, the current analysis was done on 1036 nurses. All participants declared their willingness to participate in the current study by providing a written informed consent. The study was approved by the research council and ethics committee of the School of Nutrition and Food Science, Isfahan University of Medical Sciences, Isfahan, Iran.

\section{Assessment of dietary intake}

Dietary data were collected using a Willett-format ${ }^{(16)}$, dishbased, 106-item semi-quantitative FFQ that was designed and validated specifically for Iranian adults. Detailed information about the design, the foods included ${ }^{(17)}$ as well as the validity of this questionnaire to assess the association between food consumption and $\mathrm{MetS}^{(18)}$ has been reported elsewhere. Briefly, the questionnaire contained five categories of foods and dishes: (i) mixed dishes (cooked or canned, twenty-nine items); (ii) starchy foods (different types of bread, cakes, biscuits and potato, ten items); (iii) dairy products (dairies, butter and cream, nine items); (iv) fruits and vegetables (twenty-two items); and (v) miscellaneous food items and beverages (including sweets, fast foods, nuts, desserts and beverages, thirty-six items). To develop the questionnaire, a comprehensive list of foods and dishes commonly consumed by Iranian adults was constructed. Then, we chose those foods that were nutrient-rich, consumed reasonably often or contributed to between-person variations. This process led to the remaining of the 106 food items in the questionnaire. The portion size for food items and mixed dishes was defined based on the most commonly consumed portion size for each item in the general population. To increase precision and accuracy of estimates, we attempted to give the portion size of foods and mixed dishes as a unit with the same perception for all people. Participants were asked to report their dietary intakes of foods and mixed dishes based on nine multiple-choice frequency response categories varying from 'never or less than once a month' to ' 12 or more times per day'. The number of frequency response categories was not constant for all foods. For foods consumed infrequently, we omitted the high frequency categories, while for common foods with a high consumption, the number of multiple-choice categories increased. The frequency response categories for the food list varied from six to nine choices. For instance, the frequency response for tuna consumption included six categories, as follows: never or less 
than once per month, 1-3 times/month, once per week, 2-4 times/week, 5-6 times/week, 1-2 times/d; and for tea consumption the frequency response included nine categories, as follows: never or less than 1 cup/month, 1-3 cups/month, 1-3 cups/week, 4-6 cups/week, 1 cup/d, 2-4 cups/d, 5-7 cups/d, 8-11 cups/d, $\geq 12 \mathrm{cups} / \mathrm{d}$. Finally, we computed daily intakes of all food items and then converted to grams per day using household measures ${ }^{(19)}$. Daily intakes of nutrients for each participant were calculated using the US Department of Agriculture's national nutrient databank. Total energy intake was calculated by summing up energy intakes from all foods. Total weight of foods consumed by participants was calculated by summing up the weights of foods only. We did not consider weight of drinks consumed because findings from available publications supporting the effect of DED on body weight are based on changes in weight of food intake, not drinks $^{(20)}$. In a systematic review Johnson et al. also concluded that focusing on weight of food consumed reduces the variability in the results and facilitates data interpretation $^{(21)}$. To calculate DED, we divided each individual's reported daily energy intake $(\mathrm{kcal} / \mathrm{d})$ by the total weight of foods consumed $(\mathrm{g} / \mathrm{d})$.

\section{Assessment of biochemical indicators}

To quantify biomarkers of inflammation and metabolic profile, we collected blood samples after a $12 \mathrm{~h}$ overnight fast. Fasting plasma glucose was assessed on the day of blood sampling via an enzymatic colorimetric method. Serum TAG levels were measured using enzymatic colorimetric tests with glycerol phosphate. HDL cholesterol (HDL-C) concentrations were determined after precipitation of the apo B-containing lipoproteins with phosphotungstic acid. All measurements were done using commercially available enzymatic reagents (Pars Azmoon, Tehran, Iran), adopted to an auto-analyser system (Selectra E; Vitalab, Holliston, the Netherlands). Serum concentrations of IL-6, IL-2, TNF- $\alpha$ and amyloid A were determined with the use of ELISA by means of commercially available kits (Bender MedSystems and Biosource International, Vienna, Austria). hs-CRP was assayed by ultra-sensitive latex-enhanced immunoturbidimetric assay (Randox Laboratories, Crumlin, $\mathrm{UK})$. Intra- and inter-assay $\mathrm{CV}$ for all biochemical measurements were less than $10 \%$.

\section{Assessment of blood pressure and other variables}

To evaluate blood pressure, we first asked participants to rest for $10 \mathrm{~min}$. Blood pressure was then measured using a standard mercury sphygmomanometer, twice with a 5 min interval, while participants were sitting. The mean of the two measurements was considered as the participant's blood pressure. Physical activity was assessed by use of the short-form International Physical Activity Questionnaire and expressed as metabolic equivalent-hours/ week (MET-h/week). Information about age, smoking habits, socio-economic status, medical history, menopause status and current use of medications was obtained using pre-tested questionnaires. Height, weight and waist circumference were measured based on standard protocols $^{(22)}$. BMI was calculated as weight (in kilograms) divided by the square of height (in metres).

\section{Definition of terms}

Obesity was defined as BMI $\geq 30 \cdot 0 \mathrm{~kg} / \mathrm{m}^{2}$. In the current study, MetS was defined as the presence of at least three components of the following abnormalities, according to the National Cholesterol Education Program Adult Treatment Panel III (ATP III definition) ${ }^{\text {(23) }}$ : (i) abdominal obesity (waist circumference $>88 \mathrm{~cm}$ ); (ii) low HDL-C concentration $(<50 \mathrm{mg} / \mathrm{dl})$; (iii) high serum TAG ( $\geq 150 \mathrm{mg} / \mathrm{dl}$ ); (iv) abnormal glucose homeostasis (fasting plasma glucose $>100 \mathrm{mg} / \mathrm{dl}$ ); and (v) elevated blood pressure (systolic blood pressure $\geq 130 \mathrm{mmHg}$ or diastolic blood pressure $\geq 85 \mathrm{mmHg}$ ).

\section{Statistical analysis}

Participants were categorized based on quartile cut-off points of DED. Higher quartiles of DED indicate higher energy density than lower quartiles. General characteristics of study participants across quartiles of DED were examined using ANOVA for continuous variables or the $\chi^{2}$ test for categorical variables. Age- and energy-adjusted intakes and food and nutrient consumption across quartiles of DED were calculated using ANCOVA. To determine the association between DED and MetS and its components, we used multivariable logistic regression analysis in different models. First, we controlled for the confounding effect of age. In the second model, we further adjusted for cigarette smoking (yes or no), physical activity (MET-h/week), socio-economic status (categorical), current oestrogen use (yes or no), menopausal status (yes or no) and family history of diabetes and stroke (yes or no). Further statistical control was performed for BMI (continuous) in the last model. The overall trend of odds ratios across quartiles of DED was calculated by considering the median of DED in each quartile as a continuous variable.

Due to the skewness in the distribution of inflammatory markers, all biomarkers of inflammation were first logarithmically transformed. Then, geometric means of inflammatory markers across quartiles of DED were estimated by ANCOVA in several models (covariates were same as above). All statistical analyses were performed by using the statistical software package PASW Statistics Version 18.0. $P<0.05$ was considered significant.

\section{Results}

General characteristics of the study participants across quartiles of DED are shown in Table 1. Participants with higher DED were more likely to be older, obese and 
Table 1 General characteristics by quartile of dietary energy density among female nurses $(n 1036)$ aged $>30$ years, Isfahan, Iran

\begin{tabular}{|c|c|c|c|c|c|c|c|c|c|}
\hline & \multicolumn{8}{|c|}{ Quartile of dietary energy density } & \multirow[b]{3}{*}{$P^{\star}$} \\
\hline & \multicolumn{2}{|c|}{1 (lowest) } & \multicolumn{2}{|c|}{2} & \multicolumn{2}{|c|}{3} & \multicolumn{2}{|c|}{4 (highest) } & \\
\hline & Mean & SD & Mean & SD & Mean & SD & Mean & SD & \\
\hline$n$ & \multicolumn{2}{|c|}{254} & \multicolumn{2}{|c|}{257} & \multicolumn{2}{|c|}{251} & \multicolumn{2}{|c|}{253} & \\
\hline Age (years) & 34.9 & 6.5 & $36 \cdot 8$ & 7 & $37 \cdot 8$ & $7 \cdot 2$ & 38.7 & $7 \cdot 2$ & $<0.001$ \\
\hline $\mathrm{BMI}\left(\mathrm{kg} / \mathrm{m}^{2}\right)$ & $22 \cdot 8$ & 1.6 & 23.5 & 1.9 & $24 \cdot 7$ & 1.9 & $26 \cdot 2$ & 1.7 & $<0.001$ \\
\hline WHR & 0.79 & 0.01 & 0.83 & 0.01 & 0.89 & 0.01 & 0.93 & 0.01 & $<0.001$ \\
\hline Fasting plasma glucose $(\mathrm{mmol} / \mathrm{l})$ & $5 \cdot 28$ & 0.61 & $6 \cdot 28$ & 0.67 & $6 \cdot 61$ & 0.72 & $7 \cdot 0$ & 0.83 & $<0.001$ \\
\hline TAG (mmol/l) & 1.31 & 0.26 & 1.57 & 0.28 & 1.68 & 0.32 & 2.02 & 0.20 & $<0.001$ \\
\hline Total cholesterol $(\mathrm{mmol} / \mathrm{l})$ & 3.85 & 0.88 & 4.63 & 1.01 & $5 \cdot 12$ & 1.09 & $6 \cdot 39$ & 1.32 & $<0.001$ \\
\hline LDL-C (mmol/l) & 2.35 & 0.15 & 3.08 & 0.18 & 3.33 & 0.23 & 4.09 & 0.23 & $<0.001$ \\
\hline $\mathrm{HDL}-\mathrm{C}(\mathrm{mmol} / \mathrm{l})$ & $1 \cdot 16$ & $0 \cdot 10$ & 1.14 & 0.13 & 1.01 & 0.08 & 0.80 & 0.08 & $<0.001$ \\
\hline Physical activity (MET-h/week) & $13 \cdot 2$ & 2.8 & 14.5 & 2.9 & $11 \cdot 2$ & 2.5 & $15 \cdot 3$ & 2.4 & $<0.001$ \\
\hline Family history of diabetes (\%) & \multicolumn{2}{|c|}{2} & \multicolumn{2}{|c|}{4} & \multicolumn{2}{|c|}{3} & \multicolumn{2}{|c|}{5} & 0.04 \\
\hline Family history of stroke (\%) & \multicolumn{2}{|c|}{1} & \multicolumn{2}{|c|}{1} & \multicolumn{2}{|c|}{2} & \multicolumn{2}{|c|}{1} & 0.69 \\
\hline Current daily smokers (\%) & \multicolumn{2}{|c|}{1} & \multicolumn{2}{|c|}{1} & \multicolumn{2}{|c|}{1} & \multicolumn{2}{|c|}{1} & 0.85 \\
\hline High socio-economic statust & \multicolumn{2}{|c|}{27} & \multicolumn{2}{|c|}{33} & \multicolumn{2}{|c|}{42} & \multicolumn{2}{|c|}{31} & 0.03 \\
\hline Obese $(\%) \ddagger$ & \multicolumn{2}{|c|}{13} & \multicolumn{2}{|c|}{16} & \multicolumn{2}{|c|}{21} & \multicolumn{2}{|c|}{29} & 0.01 \\
\hline Current oestrogen use (\%) & \multicolumn{2}{|c|}{8} & & & & & & & 0.22 \\
\hline Postmenopausal (\%) & & & & & & & & & 0.37 \\
\hline Metabolic syndrome (\%)§ & & & & & & & & & $<0.001$ \\
\hline Features of metabolic syndrome§ & & & & & & & & & \\
\hline Abdominal adiposity (\%) & & & & & & & & & $<0.001$ \\
\hline Elevated blood pressure (\%) & & & & & & & & & $<0.001$ \\
\hline High serum TAG (\%) & & & & & & & & & 0.01 \\
\hline Low serum HDL-C (\%) & & & & & & & & & $<0.001$ \\
\hline Abnormal glucose homeostasis (\%) & & & & & & & & & 0.11 \\
\hline
\end{tabular}

WHR, waist-to-hip ratio; LDL-C, LDL cholesterol; HDL-C, HDL cholesterol; MET, metabolic equivalent of task.

Data are presented as means and standard deviations unless indicated otherwise.

${ }^{*}$ By using linear regression.

†High socio-economic status was defined based on educational level, income, family size, being owner of the house or renting the house, and house area. ¥Obesity: BMI $\geq 30.0 \mathrm{~kg} / \mathrm{m}^{2}$.

$\S$ Metabolic syndrome was defined as the presence of three or more of the following components: (i) abdominal adiposity (waist circumference $>88 \mathrm{~cm}$ ); (ii) low serum HDL-C ( $<50 \mathrm{mg} / \mathrm{dl})$; (iii) high serum TAG ( $\geq 150 \mathrm{mg} / \mathrm{dl})$; (iv) elevated blood pressure ( $\geq 130 / 85 \mathrm{mmHg})$; (v) abnormal glucose homeostasis (fasting plasma glucose $\geq 110 \mathrm{mg} / \mathrm{dl})$.

physically active $(P<0 \cdot 001$ for all). Prevalence of MetS and its components including central obesity, elevated blood pressure, hypertriacylglycerolaemia and low HDL-C were significantly higher in the top quartile of DED compared with the bottom quartile $(P<0.001$ for all except high serum TAG, where $P=0 \cdot 01$ ). No other significant differences were seen in the general characteristics when comparing the quartiles of DED.

Age- and energy-adjusted dietary intakes of participants across quartiles of DED are presented in Table 2. As expected, individuals in the highest quartile of DED had significantly higher intakes of energy $(P=0 \cdot 005)$, fat $(P=0.001)$, refined grains, and meat and fish $(P<0 \cdot 001)$. In contrast, they had lower intakes of carbohydrate $(P=0 \cdot 01)$, fibre, whole grains, fruits, vegetables, and nuts and legumes $(P<0 \cdot 001)$. Dietary intakes of other foods and nutrients were not significantly different across quartiles of DED.

Crude and multivariable-adjusted odds ratios for MetS across quartiles of DED are provided in Table 3. After accounting for potential confounders, we found that participants in the top quartile of DED had greater odds for MetS (OR $=1 \cdot 78 ; 95 \%$ CI 1.36, 2.98) compared with those in the bottom quartile. Further control for BMI attenuated this association slightly (OR $=1 \cdot 59 ; 95 \%$ CI $1 \cdot 19,2 \cdot 81)$.
Multivariable-adjusted odds ratios for components of the MetS across quartiles of DED are indicated in Table 4. After adjustment for potential confounders, individuals in the highest quartile of DED were more likely to be abdominally obese $(\mathrm{OR}=1.51 ; 95 \%$ CI 1.00, 2.63), have hypertriacylglycerolaemia $(\mathrm{OR}=2.95 ; 95 \% \mathrm{CI} 1.58,3.91)$ and low HDL-C levels (OR $=1.36$; $95 \%$ CI $1.17,2.54)$ compared with those in the lowest quartile. Consumption of energy-dense diets was not significantly associated with the odds of abnormal glucose homeostasis or elevated blood pressure either before or after controlling for confounding variables.

Geometric means of inflammatory markers across quartiles of DED are shown in Table 5. Compared with those in the lowest quartile of DED, participants in the highest quartile had greater concentrations of hs-CRP, TNF- $\alpha$ and IL- 6 , even after adjustment for potential confounders including BMI. Mean plasma hs-CRP concentrations across increasing quartiles of DED were $1 \cdot 7,1 \cdot 7,2 \cdot 0$, $2.4 \mathrm{mg} / \mathrm{l}(P$ for trend $=0.04)$. Such increasing concentrations across increasing quartiles of DED were also seen for TNF- $\alpha(4 \cdot 1,4.5,4.5,4.8 \mathrm{ng} / 1 ; P$ for trend $=0.03)$ and IL-6 $(1 \cdot 6,1 \cdot 6,1 \cdot 5,2.5 \mathrm{ng} / \mathrm{l} ; P$ for trend $<0 \cdot 01)$. We found no significant association between consumption of 
Table 2 Dietary intakes by quartile of dietary energy density among female nurses $(n$ 1036) aged $>30$ years, Isfahan, Iran

\begin{tabular}{|c|c|c|c|c|c|c|c|c|c|}
\hline & \multicolumn{8}{|c|}{ Quartile of dietary energy density } & \multirow[b]{3}{*}{$P$} \\
\hline & \multicolumn{2}{|c|}{1 (lowest) } & \multicolumn{2}{|c|}{2} & \multicolumn{2}{|c|}{3} & \multicolumn{2}{|c|}{4 (highest) } & \\
\hline & Mean & SE & Mean & SE & Mean & $\mathrm{SE}$ & Mean & SE & \\
\hline$n$ & \multicolumn{2}{|c|}{254} & \multicolumn{2}{|c|}{257} & \multirow{2}{*}{\multicolumn{2}{|c|}{251}} & \multicolumn{2}{|c|}{253} & \\
\hline Nutrients & & & & & & & & & \\
\hline Total energy $(\mathrm{kJ} / \mathrm{d})$ & 8293 & 1360 & 8414 & 1238 & 9560 & 1305 & 9707 & 1527 & 0.005 \\
\hline Total energy $(\mathrm{kcal} / \mathrm{d})$ & 1982 & 325 & 2011 & 296 & 2285 & 312 & 2320 & 365 & 0.005 \\
\hline Carbohydrate $(\mathrm{g} / \mathrm{d})$ & 235 & 76 & 226 & 94 & 222 & 56 & 212 & 49 & 0.01 \\
\hline Protein (g/d) & 71 & 21 & 75 & 26 & 76 & 22 & 74 & 25 & 0.39 \\
\hline Fat $(\mathrm{g} / \mathrm{d})$ & 82 & 38 & 85 & 35 & 97 & 40 & 113 & 36 & 0.001 \\
\hline Cholesterol $(\mathrm{mg} / \mathrm{d})$ & 232 & 127 & 238 & 119 & 246 & 119 & 258 & 127 & 0.59 \\
\hline Dietary fibre $(\mathrm{g} / \mathrm{d})$ & 19 & 9 & 18 & 8 & 13 & 6 & 8 & 6 & $<0.001$ \\
\hline \multicolumn{10}{|l|}{ Food groups $(\mathrm{g} / \mathrm{d})$} \\
\hline Fruits & 253 & 81 & 224 & 57 & 177 & 88 & 145 & 69 & $<0.001$ \\
\hline Vegetables & 238 & 86 & 279 & 74 & 225 & 189 & 174 & 89 & $<0.001$ \\
\hline Meat and fish & 136 & 90 & 131 & 90 & 135 & 87 & 157 & 90 & $<0.001$ \\
\hline Whole grains & 99 & 25 & 72 & 24 & 53 & 28 & 23 & 25 & $<0.001$ \\
\hline Refined grains & 323 & 149 & 375 & 150 & 393 & 159 & 431 & 156 & $<0.001$ \\
\hline Dairy & 252 & 42 & 257 & 50 & 265 & 58 & 284 & 85 & 0.35 \\
\hline Nuts and legumes & 71 & 24 & 61 & 23 & 48 & 24 & 35 & 23 & $<0.001$ \\
\hline
\end{tabular}

Data are presented as means with their standard errors adjusted for age and energy intake. Data for energy intake have just been adjusted for age.

Table 3 Multivariable-adjusted odds ratios and $95 \%$ confidence intervals for metabolic syndrome across quartile of dietary energy density among female nurses $(n$ 1036) aged $>30$ years, Isfahan, Iran

\begin{tabular}{|c|c|c|c|c|c|c|c|c|}
\hline & \multicolumn{7}{|c|}{ Quartile of dietary energy density } & \multirow[b]{3}{*}{$P$ for trend ${ }^{*}$} \\
\hline & \multirow[b]{2}{*}{1 (lowest) } & \multicolumn{2}{|c|}{2} & \multicolumn{2}{|c|}{3} & \multicolumn{2}{|c|}{4 (highest) } & \\
\hline & & OR & $95 \% \mathrm{Cl}$ & OR & $95 \% \mathrm{Cl}$ & OR & $95 \% \mathrm{Cl}$ & \\
\hline$n$ & 254 & \multicolumn{2}{|c|}{257} & \multicolumn{2}{|c|}{251} & \multicolumn{2}{|c|}{253} & \\
\hline \multicolumn{9}{|c|}{ Metabolic syndrome $†$} \\
\hline Crude & 1.00 & 1.25 & $0.75,2.46$ & 1.79 & $1 \cdot 11,3.25$ & 1.96 & $1 \cdot 52,3 \cdot 11$ & $<0.001$ \\
\hline Model I & 1.00 & $1 \cdot 21$ & $0.76,2.41$ & 1.71 & $1 \cdot 10,3 \cdot 21$ & 1.90 & $1.48,3.07$ & $<0.001$ \\
\hline Model II§ & 1.00 & $1 \cdot 14$ & $0.82,2.28$ & 1.65 & $1 \cdot 07,3 \cdot 11$ & 1.78 & $1.36,2.98$ & $<0.001$ \\
\hline Model IIIII & 1.00 & 1.02 & $0.77,2.01$ & 1.54 & $1.01,2.89$ & 1.59 & $1 \cdot 19,2 \cdot 81$ & $<0.001$ \\
\hline
\end{tabular}

*By the use of Mantel-Haenszel extension $x^{2}$ test.

†Metabolic syndrome was defined as the presence of three or more of the following components: (i) abdominal adiposity (waist circumference $>88 \mathrm{~cm}$ ); (ii) low serum HDL cholesterol ( $<50 \mathrm{mg} / \mathrm{dl}$ ); (iii) high serum TAG ( $\geq 150 \mathrm{mg} / \mathrm{dl}$ ); (iv) elevated blood pressure ( $\geq 130 / 85 \mathrm{mmHg}$ ); (v) abnormal glucose homeostasis (fasting plasma glucose $\geq 110 \mathrm{mg} / \mathrm{dl}$ ).

$\ddagger$ Adjusted for age.

§Further adjusted for cigarette smoking, physical activity, socio-economic status, current oestrogen use, menopausal status and family history of diabetes and stroke.

IAdditionally adjusted for BMI.

energy-dense diets and serum concentrations of amyloid A and IL-2 either in crude or adjusted models.

\section{Discussion}

In the current cross-sectional study, DED was positively associated with MetS and features of MetS including abdominal adiposity, low HDL-C levels and hypertriacylglycerolaemia in Iranian female nurses. Additionally, individuals in the top quartile of DED had higher plasma concentrations of hs-CRP, TNF- $\alpha$ and IL- 6 . All associations were independent of BMI.

Despite a growing interest in evaluating health outcomes related to DED in nutritional epidemiology, there is still no standardized calculation method. The major controversy is related to including or excluding beverages in the calculations. The most common method in epidemiological studies is using foods only, excluding beverages, since it has been suggested this method could better demonstrate the meaning of $\operatorname{DED}^{(8,21,24)}$. Therefore, in the present study, we included only foods, not beverages, in DED calculation.

A growing body of evidence has demonstrated adverse effects of high-energy-dense diets on insulin resistance, metabolic profile and anthropometric measures. Therefore, as expected, high DED was associated with increased risk of MetS. This was in line with previous epidemiological studies ${ }^{(10,12,24)}$. This finding concurred with the results of a cross-sectional study among American adults, where Mendoza et $a l^{(24)}$ demonstrated that higher DED is 
Table 4 Multivariable-adjusted odds ratios and $95 \%$ confidence intervals for components of the metabolic syndrome* across quartile of dietary energy density among female nurses $(n$ 1036) aged $>30$ years, Isfahan, Iran

\begin{tabular}{|c|c|c|c|c|c|c|c|c|}
\hline & \multicolumn{7}{|c|}{ Quartile of dietary energy density } & \multirow[b]{3}{*}{$P$ for trend $\dagger$} \\
\hline & \multirow[b]{2}{*}{1 (lowest) } & \multicolumn{2}{|c|}{2} & \multicolumn{2}{|c|}{3} & \multicolumn{2}{|c|}{4 (highest) } & \\
\hline & & OR & $95 \% \mathrm{Cl}$ & OR & $95 \% \mathrm{Cl}$ & OR & $95 \% \mathrm{Cl}$ & \\
\hline$n$ & 254 & \multicolumn{2}{|c|}{257} & \multicolumn{2}{|c|}{251} & \multicolumn{2}{|c|}{253} & \\
\hline \multicolumn{9}{|c|}{ Abdominal adiposity } \\
\hline Crude & 1.00 & 1.17 & $0.75,1.95$ & 1.45 & $0.85,1.92$ & 1.76 & $1.02,2.91$ & $<0.001$ \\
\hline Model I & 1.00 & $1 \cdot 14$ & $0.71,1.84$ & 1.41 & $0.87,1.90$ & 1.69 & $1 \cdot 05,2 \cdot 81$ & \\
\hline Model II§ & 1.00 & 1.09 & $0.68,1.89$ & 1.35 & $0.82,1.81$ & 1.60 & $1.03,2.74$ & \\
\hline Model IIIII & 1.00 & 1.01 & $0.61,1.80$ & 1.28 & $0.77,1.83$ & 1.51 & $1.00,2.63$ & \\
\hline \multicolumn{9}{|c|}{ Elevated blood pressure } \\
\hline Crude & 1.00 & 1.06 & $0.69,1.45$ & 1.22 & $0.73,1.85$ & 1.42 & $0.91,1.99$ & $<0.001$ \\
\hline Model I & 1.00 & 1.03 & $0.61,1.41$ & 1.19 & $0.71,1.80$ & 1.37 & $0.89,1.95$ & \\
\hline Model II & 1.00 & 0.98 & $0.62,1.44$ & $1 \cdot 12$ & $0.65,1.81$ & 1.34 & $0.86,1.91$ & \\
\hline Model III & 1.00 & 0.94 & $0.58,1.38$ & $1 \cdot 12$ & $0.67,1.75$ & 1.20 & $0.80,1.84$ & \\
\hline \multicolumn{9}{|c|}{ High serum TAG } \\
\hline Crude & 1.00 & 1.45 & $0.97,2.22$ & $2 \cdot 26$ & $1 \cdot 17,2 \cdot 97$ & $3 \cdot 25$ & $1.67,4.20$ & $<0.001$ \\
\hline Model I & 1.00 & 1.40 & $0.92,1.20$ & $2 \cdot 20$ & $1 \cdot 15,2.94$ & 3.19 & $1.68,4.09$ & \\
\hline Model II & 1.00 & 1.37 & $0.94,1.17$ & $2 \cdot 12$ & $1 \cdot 10,2.90$ & 3.08 & $1.64,4.00$ & \\
\hline Model III & 1.00 & 1.24 & $0.88,1.11$ & $2 \cdot 01$ & $1.02,2 \cdot 81$ & 2.95 & $1.58,3.91$ & \\
\hline \multicolumn{9}{|c|}{ Low serum HDL cholesterol } \\
\hline Crude & 1.00 & 1.29 & $1 \cdot 02,2 \cdot 21$ & 1.42 & $1 \cdot 11,2 \cdot 31$ & 1.56 & $1.25,2.71$ & $<0.001$ \\
\hline Model I & 1.00 & $1 \cdot 21$ & $0.99,2.17$ & 1.36 & $1 \cdot 08,2 \cdot 27$ & 1.50 & $1.21,2.67$ & \\
\hline Model II & 1.00 & 1.13 & $0.94,2.11$ & 1.37 & $1 \cdot 05,2 \cdot 18$ & 1.42 & $1 \cdot 19,2.60$ & \\
\hline Model III & 1.00 & 1.07 & $0.91,2.04$ & 1.31 & $1 \cdot 07,2 \cdot 15$ & 1.36 & $1.17,2.54$ & \\
\hline \multicolumn{9}{|c|}{ Abnormal glucose homeostasis } \\
\hline Crude & 1.00 & 1.22 & $0.59,3 \cdot 19$ & 1.21 & $0.59,3.01$ & 1.41 & $0.62,2.96$ & 0.55 \\
\hline Model I & 1.00 & 1.20 & $0.57,3 \cdot 11$ & 1.19 & $0.61,2.96$ & 1.38 & $0.60,2.90$ & \\
\hline Model II & 1.00 & 1.14 & $0.58,3.04$ & $1 \cdot 15$ & $0.58,2.91$ & 1.35 & $0.61,2.85$ & \\
\hline Model III & 1.00 & 1.07 & $0.53,2.96$ & 1.06 & $0.51,2.84$ & 1.28 & $0.65,2.78$ & \\
\hline
\end{tabular}

${ }^{*}$ Components of the metabolic syndrome were defined as follows: abdominal adiposity (waist circumference $>88 \mathrm{~cm}$ ); low serum $\mathrm{HDL}$ cholesterol $(<50 \mathrm{mg} / \mathrm{dl}$ ); high serum TAG ( $\geq 150 \mathrm{mg} / \mathrm{dl})$; elevated blood pressure $(\geq 130 / 85 \mathrm{mmHg})$; abnormal glucose homeostasis (fasting plasma glucose $\geq 110 \mathrm{mg} / \mathrm{dl})$.

†By the use of Mantel-Haenszel extension $x^{2}$ test.

$\ddagger$ Adjusted for age.

§Further adjusted for cigarette smoking, physical activity, socio-economic status, current oestrogen use, menopausal status and family history of diabetes and stroke.

IIAdditionally adjusted for BMI.

significantly associated with higher risk of MetS. In the current study, dietary data from a validated FFQ were used; however, the findings were similar to those of Mendoza et $a l .{ }^{(24)}$ in spite of the use of data from one-day $24 \mathrm{~h}$ dietary recall in their study. DED provides an overall picture of diet since it considers all foods together. Nevertheless, it has its own characteristics in each population and might lead to different and specific outcomes. For example, in the Mediterranean dietary pattern, despite the higher content of fat, an inverse correlation has been reported between olive oil consumption and DED. This might be attributed to consuming olive oil along with vegetables in salad. Further studies are required to clarify the associations between DED and metabolic abnormalities in different populations with different dietary patterns. Adverse effects of energy-dense diets on metabolic profile and inflammatory markers might be explained by their unhealthy components such as refined grains, trans- and saturated fatty acids, and processed and red meats. Furthermore, higher-energy-dense diets are associated with greater energy intake ${ }^{(8)}$, but lower dietary diversity score, which leads to lower intakes of fruit, vegetables and whole grains ${ }^{(10,25)}$. Altogether, these dietary factors may lead to increased adiposity, particularly abdominal adiposity, and insulin resistance, as components of $\mathrm{MetS}^{(26-29)}$. On the other hand, regarding the poor diet quality of Iranians ${ }^{(30,31)}$, lower-energy-dense diets may ameliorate metabolic abnormalities by improving diet quality.

The possible mechanisms underlying the association of DED and inflammation are not well understood. Earlier studies have indicated that consuming a more energydense diet is associated with weight gain ${ }^{(6,32)}$ and greater adiposity tissue ${ }^{(33)}$. Adipose tissue is the main source of inflammatory biomarkers, and therefore this might be the link between DED and higher inflammatory biomarkers. However, even after adjustment for BMI, this association remained significant. It should be kept in mind that there is not a simple linear relationship between BMI and abdominal adiposity, as a component of MetS, and the main source of inflammatory biomarkers. It is possible that the saturated fat, cholesterol and refined carbohydrate contents of high-energy-dense diets, which are positively correlated with dietary inflammatory index ${ }^{(34)}$, contribute to its relationship with chronic inflammation status.

The lack of association between DED and abnormal glucose homeostasis in the current study is in line with 
Table 5 Geometric means of inflammatory markers across quartile of dietary energy density among female nurses ( $n$ 1036) aged $>30$ years, Isfahan, Iran

\begin{tabular}{|c|c|c|c|c|c|c|c|c|c|}
\hline & \multicolumn{8}{|c|}{ Quartile of dietary energy density } & \multirow[b]{3}{*}{$P^{\star}$} \\
\hline & \multicolumn{2}{|c|}{1 (lowest) } & \multicolumn{2}{|c|}{2} & \multicolumn{2}{|c|}{3} & \multicolumn{2}{|c|}{4 (highest) } & \\
\hline & Mean & SE & Mean & SE & Mean & SE & Mean & SE & \\
\hline \multicolumn{10}{|c|}{ hs-CRP (mg/l) } \\
\hline Crude & 1.9 & 1.8 & $2 \cdot 1$ & $1 \cdot 8$ & $2 \cdot 6$ & 1.9 & $3 \cdot 2$ & $2 \cdot 0$ & $<0.01$ \\
\hline Model I† & $1 \cdot 8$ & 1.7 & $2 \cdot 0$ & 1.7 & $2 \cdot 6$ & $1 \cdot 8$ & $3 \cdot 1$ & 1.9 & $<0.01$ \\
\hline Model II‡ & 1.9 & 1.5 & 1.9 & 1.5 & $2 \cdot 2$ & $1 \cdot 7$ & $2 \cdot 8$ & $1 \cdot 8$ & 0.02 \\
\hline Model III§ & $1 \cdot 7$ & 1.6 & 1.7 & 1.6 & $2 \cdot 0$ & $1 \cdot 6$ & $2 \cdot 4$ & $1 \cdot 7$ & 0.04 \\
\hline \multicolumn{10}{|l|}{ TNF-a (ng/l) } \\
\hline Crude & $3 \cdot 8$ & $2 \cdot 1$ & 4.2 & $2 \cdot 2$ & 4.6 & $2 \cdot 1$ & $5 \cdot 3$ & $2 \cdot 1$ & $<0.01$ \\
\hline Model I & 3.9 & $2 \cdot 0$ & $4 \cdot 1$ & $2 \cdot 1$ & 4.4 & $2 \cdot 0$ & $5 \cdot 4$ & $2 \cdot 0$ & $<0.01$ \\
\hline Model II & 3.7 & $1 \cdot 8$ & $4 \cdot 3$ & $2 \cdot 0$ & $4 \cdot 3$ & $2 \cdot 0$ & $5 \cdot 2$ & $1 \cdot 8$ & 0.01 \\
\hline Model III & $4 \cdot 1$ & 1.5 & 4.5 & 1.9 & 4.5 & $2 \cdot 2$ & $4 \cdot 8$ & $1 \cdot 8$ & 0.03 \\
\hline \multicolumn{10}{|l|}{ IL-6 (ng/l) } \\
\hline Crude & $1 \cdot 3$ & 1.0 & $1 \cdot 7$ & 1.5 & $2 \cdot 1$ & 1.9 & $2 \cdot 9$ & $2 \cdot 1$ & $<0.01$ \\
\hline Model I & $1 \cdot 2$ & 1.0 & 1.5 & 1.5 & $2 \cdot 0$ & $1 \cdot 8$ & $2 \cdot 8$ & $2 \cdot 0$ & $<0.01$ \\
\hline Model II & 1.4 & $1 \cdot 1$ & 1.4 & $1 \cdot 6$ & $1 \cdot 8$ & $1 \cdot 8$ & $2 \cdot 8$ & $1 \cdot 8$ & $<0.01$ \\
\hline Model III & $1 \cdot 6$ & 1.2 & 1.6 & 1.4 & 1.5 & $1 \cdot 7$ & 2.5 & $1 \cdot 7$ & $<0.01$ \\
\hline \multicolumn{10}{|l|}{ SAA (mg/l) } \\
\hline Crude & 4.5 & $2 \cdot 7$ & 4.4 & $2 \cdot 7$ & $4 \cdot 8$ & $2 \cdot 0$ & $4 \cdot 3$ & $2 \cdot 1$ & 0.35 \\
\hline Model I & $4 \cdot 3$ & 2.5 & 4.4 & 2.5 & $4 \cdot 6$ & $2 \cdot 0$ & $4 \cdot 2$ & $2 \cdot 0$ & 0.39 \\
\hline Model II & $4 \cdot 1$ & $2 \cdot 4$ & $4 \cdot 3$ & 2.5 & 4.5 & $2 \cdot 1$ & $4 \cdot 1$ & $2 \cdot 0$ & 0.41 \\
\hline Model III & 4.0 & $2 \cdot 2$ & 4.0 & $2 \cdot 4$ & 4.4 & 1.9 & 4.0 & 1.9 & 0.59 \\
\hline \multicolumn{10}{|l|}{ IL-2 (ng/l) } \\
\hline Crude & 1.5 & $2 \cdot 1$ & $1 \cdot 6$ & $2 \cdot 1$ & 1.4 & $1 \cdot 8$ & 1.6 & 1.5 & 0.47 \\
\hline Model I & 1.4 & $2 \cdot 0$ & 1.5 & $2 \cdot 1$ & 1.4 & 1.7 & 1.6 & 1.4 & 0.42 \\
\hline Model II & $1 \cdot 3$ & $2 \cdot 0$ & 1.4 & 1.9 & 1.6 & 1.6 & 1.5 & 1.4 & 0.57 \\
\hline Model III & $1 \cdot 3$ & 1.8 & 1.6 & $1 \cdot 8$ & 1.5 & 1.6 & 1.4 & $1 \cdot 3$ & 0.63 \\
\hline
\end{tabular}

hs-CRP, high-sensitivity C-reactive protein; SAA, serum amyloid A.

Data are presented as geometric means with their standard errors,

${ }^{*}$ By the use of ANOVA in crude models and ANCOVA in the adjusted models.

†Adjusted for age.

‡Further adjusted for cigarette smoking, physical activity, socio-economic status, current oestrogen use, menopausal status and family history of diabetes and stroke.

§Additionally adjusted for BMI.

earlier investigations ${ }^{(4,12,14)}$. The possible explanation for this finding might be the lower prevalence of abnormal glucose homeostasis in our study population. Another explanation might be the quality of fibre and fat, besides their quantity, which might differently affect glucose homeostasis ${ }^{(35-37)}$. Energy-dense diets contain greater amounts of trans and saturated fats ${ }^{(5)}$. Additionally, we have used only fasting plasma glucose to define abnormal glucose homeostasis, which might lead to the misclassification of study participants in terms of this condition. To further explore the relationship between DED and glycaemic control, one would need to consider fasting insulin levels as well.

Our study has several limitations that should be taken into account when interpreting our findings. The inherent limitation of its cross-sectional design does not allow us to draw conclusions on causality. Although having a metabolic disorder does not necessarily lead to unhealthy eating, longitudinal studies are required to investigate causal relationships. The study was conducted among female nurses, which may further limit the external validity of our findings. On the other hand, shift work and sleep deprivation in this population may affect their dietary intakes ${ }^{(38)}$ as well as their metabolic profile and weight ${ }^{(39-41)}$. Using the US Department of Agriculture's food composition table to estimate nutrient and energy intakes in this population is another limitation of the present study, although efforts have been made to adapt this software for Iranians. Our observational study was conducted among free-living individuals, which could better reflect the real associations because of considering habitual dietary intakes rather than short-term intervention. Although we controlled for several potential confounders, unknown or unmeasured confounding variables like sleep deprivation cannot be excluded. Misclassification of participants due to using a self-administered, dish-based semi-quantitative FFQ is another limitation of our study, as with all other epidemiological studies. Substantially large sample size and considering the confounding effects of various variables are the strengths of the present study.

\section{Conclusion}

In summary, we found that consumption of highenergy-dense diets was associated with increased chance of MetS and most of its features in Iranian female nurses. Additionally, DED was positively associated with 
inflammatory markers including hs-CRP, TNF- $\alpha$ and IL- 6 concentrations. Longitudinal studies are needed to explore the relationship of DED with MetS and inflammation.

\section{Acknowledgements}

Financial support: The study was supported by the Food Security Research Center, Isfahan University of Medical Sciences, Isfahan, Iran in conjunction with Lorestan University of Medical Sciences, Khorramabad, Iran. Conflict of interest: The authors declared no conflict of interest. Authorship: L.A. and A.E. were involved in conception, design, data collection and statistical analysis. F.H. was involved in data interpretation and paper drafting. B.L. was involved in paper drafting and editing. L.A. and A.E. supervised the study. Ethics of buman subject participation: This study was approved by the research council and ethics committee of the School of Nutrition and Food Science, Isfahan University of Medical Sciences, Isfahan, Iran. All participants declared their willingness to participate in the study by providing written informed consent.

\section{References}

1. O'Neill S \& O'Driscoll L (2015) Metabolic syndrome: a closer look at the growing epidemic and its associated pathologies. Obes Rev 16, 1-12.

2. Al Thani M, Al Thani AA, Al-Chetachi W et al. (2016) A 'high risk' lifestyle pattern is associated with metabolic syndrome among Qatari women of reproductive age: a cross-sectional national study. Int J Mol Sci 17, E698.

3. Kant AK \& Graubard BI (2005) Energy density of diets reported by American adults: association with food group intake, nutrient intake, and body weight. Int J Obes (Lond) 29, 950-956.

4. Vernarelli JA, Mitchell DC, Rolls BJ et al. (2015) Dietary energy density is associated with obesity and other biomarkers of chronic disease in US adults. Eur J Nutr 54, 59-65.

5. Bes-Rastrollo M, van Dam RM, Martinez-Gonzalez MA et al. (2008) Prospective study of dietary energy density and weight gain in women. Am J Clin Nutr 88, 769-777.

6. Savage JS, Marini M \& Birch LL (2008) Dietary energy density predicts women's weight change over 6 y. Am J Clin Nutr 88, 677-684.

7. Melanson KJ, Summers A, Nguyen V et al. (2012) Body composition, dietary composition, and components of metabolic syndrome in overweight and obese adults after a 12-week trial on dietary treatments focused on portion control, energy density, or glycemic index. Nutr J 11, 57.

8. Ledikwe JH, Blanck HM, Kettel Khan L et al. (2006) Dietary energy density is associated with energy intake and weight status in US adults. Am J Clin Nutr 83, 1362-1368.

9. Rouhani MH, Haghighatdoost F, Surkan PJ et al. (2016) Associations between dietary energy density and obesity: a systematic review and meta-analysis of observational studies. Nutrition 32, 1037-1047.

10. Esmaillzadeh A \& Azadbakht L (2011) Dietary energy density and the metabolic syndrome among Iranian women. Eur J Clin Nutr 65, 598-605.

11. Wang J, Luben R, Khaw KT et al. (2008) Dietary energy density predicts the risk of incident type 2 diabetes: the European Prospective Investigation of Cancer (EPIC)Norfolk Study. Diabetes Care 31, 2120-2125.
12. Esmaillzadeh A, Boroujeni HK \& Azadbakht L (2012) Consumption of energy-dense diets in relation to cardiometabolic abnormalities among Iranian women. Public Health Nutr 15, 868-875.

13. Wang J, Zhang W, Sun L et al. (2013) Dietary energy density is positively associated with risk of pancreatic cancer in urban Shanghai Chinese. J Nutr 143, 1626-1629.

14. van den Berg SW, van der AD, Spijkerman AM et al. (2013) The association between dietary energy density and type 2 diabetes in Europe: results from the EPIC-InterAct Study. PLoS One 8, e59947.

15. Tabesh M, Hosseinzadeh MJ, Tabesh M et al. (2013) Effects of dietary energy density on serum adipocytokine levels in diabetic women. Horm Metab Res 45, 834-839.

16. Willett W (2012) Nutritional Epidemiology, 3rd ed. New York: Oxford University Press.

17. Keshteli A, Esmaillzadeh A, Rajaie S et al. (2014) A dishbased semi-quantitative food frequency questionnaire for assessment of dietary intakes in epidemiologic studies in Iran: design and development. Int J Prev Med 5, 29-36.

18. Zaribaf F, Falahi E, Barak F et al. (2014) Fish consumption is inversely associated with the metabolic syndrome. Eur $J$ Clin Nutr 68, 474-480.

19. Ghaffarpour M, Houshiar-Rad A \& Kianfar H (1999) The Manual for Household Measures, Cooking Yields Factors and Edible Portion of Foods. Tehran: Nashre Olume Keshavarzy.

20. Ledikwe JH, Blanck HM, Khan LK et al. (2005) Dietary energy density determined by eight calculation methods in a nationally representative United States population. $J$ Nutr 135, 273-278.

21. Johnson L, Wilks DC, Lindroos AK et al. (2009) Reflections from a systematic review of dietary energy density and weight gain: is the inclusion of drinks valid? Obes Rev 10, 681-692.

22. Esmaillzadeh A, Mirmiran P \& Azizi F (2006) Comparative evaluation of anthropometric measures to predict cardiovascular risk factors in Tehranian adult women. Public Health Nutr 9, 61-69.

23. Expert Panel on Detection, Evaluation, and Treatment of High Blood Cholesterol in Adults (2001) Executive summary of the third report of the National Cholesterol Education Program (NCEP) Expert Panel on Detection, Evaluation, and Treatment of High Blood Cholesterol in Adults (Adult Treatment Panel III). JAMA 285, 2486-2497.

24. Mendoza JA, Drewnowski A \& Christakis DA (2007) Dietary energy density is associated with obesity and the metabolic syndrome in US adults. Diabetes Care 30, 974-979.

25. Azadbakht L \& Esmaillzadeh A (2012) Dietary energy density is favorably associated with dietary diversity score among female university students in Isfahan. Nutrition $\mathbf{2 8}$, 991-995.

26. Radhika G, Van Dam RM, Sudha V et al. (2009) Refined grain consumption and the metabolic syndrome in urban Asian Indians (Chennai Urban Rural Epidemiology Study 57). Metabolism 58, 675-681.

27. Zhou YJ, Tang YS, Song YL et al. (2013) Saturated fatty acid induces insulin resistance partially through nucleotidebinding oligomerization domain 1 signaling pathway in adipocytes. Chin Med Sci J 28, 211-2117.

28. Anderson AL, Harris TB, Tylavsky FA et al. (2012) Dietary patterns, insulin sensitivity and inflammation in older adults. Eur J Clin Nutr 66, 18-24.

29. Azadbakht L, Mirmiran P \& Azizi F (2005) Dietary diversity score is favorably associated with the metabolic syndrome in Tehranian adults. Int J Obes (Lond) 29, 1361-1367.

30. Mirmiran P, Azadbakht L \& Azizi F (2007) Dietary behaviour of Tehranian adolescents does not accord with their nutritional knowledge. Public Health Nutr 10, 897-901. 
31. Azadbakht L, Mirmiran P, Hosseini F et al. (2005) Diet quality status of most Tehranian adults needs improvement. Asia Pac J Clin Nutr 14, 163-168.

32. Vergnaud A-C, Estaquio C, Czernichow S et al. (2009) Energy density and 6-year anthropometric changes in a middle-aged adult cohort. Br J Nutr 102, 302-309.

33. Johnson L, Mander AP, Jones LR et al. (2008) A prospective analysis of dietary energy density at age 5 and 7 years and fatness at 9 years among UK children. Int J Obes (Lond) $\mathbf{3 2}$ 586-593.

34. Cavicchia PP, Steck SE, Hurley TG et al. (2009) A new dietary inflammatory index predicts interval changes in serum highsensitivity C-reactive protein. J Nutr 139, 2365-2372.

35. Sartorelli DS, Freire RD, Ferreira SR et al. (2005) Dietary fiber and glucose tolerance in Japanese Brazilians. Diabetes Care 28, 2240-2242.

36. Schwab U, Lauritzen L, Tholstrup T et al. (2014) Effect of the amount and type of dietary fat on cardiometabolic risk factors and risk of developing type 2 diabetes, cardiovascular diseases, and cancer: a systematic review. Food Nutr Res 2014, 58.
37. Bjermo H, Iggman D, Kullberg J et al. (2012) Effects of $n-6$ PUFAs compared with SFAs on liver fat, lipoproteins, and inflammation in abdominal obesity: a randomized controlled trial. Am J Clin Nutr 95, 1003-1012.

38. Haghighatdoost F, Karimi G, Esmaillzadeh A et al. (2012) Sleep deprivation is associated with lower diet quality indices and higher rate of general and central obesity among young female students in Iran. Nutrition $\mathbf{2 8}$, 1146-1150.

39. Kim MJ, Son KH, Park HY et al. (2013) Association between shift work and obesity among female nurses: Korean Nurses' Survey. BMC Public Health 13, 1204.

40. Chaput JP, McNeil J, Despres JP et al. (2013) Short sleep duration as a risk factor for the development of the metabolic syndrome in adults. Prev Med 57, 872-877.

41. Ohkuma T, Fujii H, Iwase $\mathrm{M}$ et al. (2014) U-shaped association of sleep duration with metabolic syndrome and insulin resistance in patients with type 2 diabetes: the Fukuoka Diabetes Registry. Metabolism 63, 484-491. 